\title{
BLOCK PARTITIONS: AN EXTENDED VIEW
}

\author{
I. BÁRÁNY, E. CSÓKA, GY. KÁROLYI, AND G. TÓTH
}

\begin{abstract}
Given a sequence $S=\left(s_{1}, \ldots, s_{m}\right) \in[0,1]^{m}$, a block $B$ of $S$ is a subsequence $B=$ $\left(s_{i}, s_{i+1}, \ldots, s_{j}\right)$. The size $b$ of a block $B$ is the sum of its elements. It is proved in [1] that for each positive integer $n$, there is a partition of $S$ into $n$ blocks $B_{1}, \ldots, B_{n}$ with $\left|b_{i}-b_{j}\right| \leq 1$ for every $i, j$. In this paper, we consider a generalization of the problem in higher dimensions.
\end{abstract}

\section{INTRODUCTION}

This paper is a follow-up to [1, which is about block partitions of sequences $S=\left(s_{1}, \ldots, s_{m}\right)$ of real numbers. A block $B$ of $S$ is either a sequence $B=\left(s_{i}, s_{i+1}, \ldots, s_{j}\right)$ where $i \leq j$ or the empty set. The size $b$ of a block $B$ is the sum of its elements. One of the main results of [1] says the following.

Theorem 1.1. Given a sequence $S=\left(s_{1}, \ldots, s_{m}\right)$ of real numbers with $s_{i} \in[0,1]$ for all $i$, and an integer $n \in \mathbb{N}$, there is a partition of $S$ into $n$ blocks such that $\left|b_{i}-b_{j}\right| \leq 1$ for all $i, j$.

The bound given here is best possible as shown by the example when all the $s_{i}=1$ and $n$ does not divide $m$.

Here, we rephrase or generalize the setting and the result in the following way. Define $a_{i}=\sum_{j=1}^{i} s_{j}$ (where $a_{0}=0$ ) and set $A=\left\{a_{0}, \ldots, a_{m}\right\}$. A partition of $A$ into $n$ blocks is the same as choosing indices $x_{0}=0 \leq x_{1} \leq \cdots \leq x_{n}=m$ so that $x_{j}$ is represented by $a_{x_{j}} \in A$, and then $b_{j}=a_{x_{j}}-a_{x_{j-1}}$ for all $i \in[n]$. Here $[n]$ denotes the set $\{1, \ldots, n\}$. For the more general setting, we consider closed sets $\left(A_{i}\right)=A_{0}, A_{1}, \ldots, A_{n} \subset \mathbb{R}$ satisfying the following conditions:

(i) $A_{0}=\{0\}, A_{n}=\{s\}$.

(ii) $A_{j} \cap[a, a+1] \neq \emptyset$ for every $j \in[n-1]$ and for every $a \in \mathbb{R}$.

A transversal $T=\left(a_{0}, a_{1}, \ldots, a_{n}\right)$ of the system $A_{j}$ is simply a selection of elements $a_{j} \in A_{j}$ for every $j=0,1, \ldots, n$. Given a transversal, we define $z_{j}=a_{j}-a_{j-1}$ for all $j \in[n]$. In this setting, $z_{j}$ corresponds to the size $b_{j}$ of the $j$ th block.

Theorem 1.2. Under the above conditions, there is a transversal $a_{j} \in A_{j}$ such that

$$
\left|z_{i}-z_{j}\right| \leq 1 \text { for all } i, j \in[n] .
$$

The bound $\left|z_{i}-z_{j}\right| \leq 1$ is again best possible as shown by (essentially the same) example: $A_{0}=\{0\}$, $A_{i}=\{0, \pm 1, \pm 2, \ldots\}$ for $i \in[n-1]$ and $A_{n}=\{s\}$, with an integer $s$ not divisible by $n$. Without the closedness of the sets $A_{j}$, we would only have a transversal with $\left|z_{i}-z_{j}\right| \leq 1+\varepsilon$ for each $\varepsilon>0$, as shown by the following example: let $n \geq 3, A_{j}=(\infty,-1 / 2] \cup(1 / 2, \infty)$ for odd $j, A_{j}=(\infty,-1 / 2) \cup[1 / 2, \infty)$ for even $j$.

Theorem 1.2 could be easily deduced from Theorem 1.1, but we will present a new and shorter direct proof in the next section. Then we extend the new setting to higher dimensions.

Let $B_{d}$ be the unit ball of a norm $\|\cdot\|$ in $\mathbb{R}^{d}$. Let $A_{0}, A_{1}, \ldots, A_{n}$ be a sequence of closed sets in $\mathbb{R}^{d}$. It is called a grid-like sequence if (i) $A_{0}=\{0\}$ and $A_{n}=\{s\}$ for a fixed element $s \in \mathbb{R}^{d}$, and (ii) each of $A_{1}, A_{2}, \ldots, A_{n-1}$ intersect with all unit balls, or formally, $\forall i \in[n-1], \forall a \in \mathbb{R}^{d}$ :

$$
\left(a+B_{d}\right) \cap A_{i} \neq \emptyset .
$$


Note that in the one dimensional case we required $A_{i} \cap[a, a+1] \neq \emptyset$ while the above condition would translate to $A_{i} \cap[a-1, a+1] \neq \emptyset$. So there is a factor of 2 in the new setting.

Given a transversal $T$, we define again $z_{i}=a_{i}-a_{i-1}$ for $i \in[n]$ and set $Z=\operatorname{conv}\left\{z_{1}, \ldots, z_{n}\right\}$. The goal is to find a transversal $T$ such that

$$
D(T)=D\left(T,\left(A_{i}\right)\right)=\operatorname{diam} Z=\max _{i, j}\left\|z_{i}-z_{j}\right\|
$$

is as small as possible. Let

$$
D_{d}^{*}=\sup _{\left(A_{i}\right)} \min _{T} D\left(T,\left(A_{i}\right)\right)
$$

for grid-like sequences $\left(A_{i}\right)$ and transversals $T$. Due to the closedness of each $A_{i}$, this minimum always exists. It is easy to see the following two propositions.

Proposition 1.3. $D_{d+1}^{*} \geq D_{d}^{*}$

Proof. If $A_{0}, A_{1}, \ldots, A_{n} \subset \mathbb{R}^{d}$ is a grid-like sequence, then $A_{0}, A_{1} \times \mathbb{R}, A_{2} \times \mathbb{R} \ldots A_{n-1} \times \mathbb{R}, A_{n} \subset \mathbb{R}^{d+1}$ is also a grid-like sequence, and the $n$-dimensional projection of any transversal of the latter sequence is a transversal of the former sequence with at most the same diameter.

Proposition 1.4. $D_{d}^{*} \leq 4$, or in other words, there is always a transversal $T$ with $D(T) \leq 4$.

Proof. Set $t=\frac{s}{n}$ and choose a point $a_{i} \in A_{i}$ from $i t+B$ for $i \in[n-1]$, so $a_{i}=i t+b_{i}$ with $b_{i} \in B$, for all $i=0, \ldots, n$. Then $z_{i}=t+b_{i}-b_{i-1}$, and so $z_{i}-z_{j}=b_{i}-b_{i-1}-b_{j}+b_{j-1}$, clearly in $4 B$.

One could hope that the better bound $D_{d}^{*} \leq 2$, which is valid for $d=1$, also holds in higher dimensions. But this is not the case, at least with Euclidean norm:

\section{Theorem 1.5.}

$$
D_{2}^{*} \geq 4 \sqrt{2-\sqrt{3}} \approx 2.071 .
$$

In more detail, for every $\varepsilon>0$ and $n \geq n_{0}(\varepsilon)$, there exists a grid-like sequence $A_{0}, A_{1}, \ldots, A_{n} \subset \mathbb{R}^{2}$ such that for any transversal $T, D(T) \leq 4 \sqrt{2-\sqrt{3}}$.

We have a stronger bound $1+\sqrt{2} \approx 2.414$, see Theorem 1.6 below, which may be sharp for $d=2$ or maybe even for all $d$. Its proof is based on the same ideas as that of Theorem 1.5 , but it is much longer and more complicated case analysis. Therefore, we prove Theorem 1.5 and give an informal description of the construction for Theorem 1.6, but omit the proof.

Theorem 1.6. $D_{2}^{*} \geq 1+\sqrt{2} \approx 2.414$.

Apart from the trivial bound $D_{d}^{*} \leq 4$ given in Proposition 1.4, we cannot prove any upper bound $\}_{1}^{1}$ not even in the case of the maximum norm $\|\cdot\|_{\infty}$. Note that the existence of a transversal $T$ with " $D(T) \leq 2$ in the maximum norm" would imply the bound $D_{d}^{*} \leq 2 \sqrt{2}$ (in the Euclidean norm). For some related problems and results we refer to [2].

Remark. We may assume without any loss that $s=0$. Indeed, with the previous meaning of $t$, set $A_{i}^{*}=A_{i}-i t$. Then the system $A_{i}^{*}$ with $s^{*}=0$ satisfies condition (1) and it is easy to check that for the transversals $a_{i} \in A_{i}$ and $a_{i}^{*}=a_{i}-i t \in A_{i}^{*}$ one has the same $z_{i}-z_{j}=z_{i}^{*}-z_{j}^{*}$ and then the diameters of $Z$ and $Z^{*}$ coincide. Note that $0=\frac{1}{n} \sum_{i=1}^{n} z_{i}^{*} \in Z^{*}$.

\footnotetext{
${ }^{1}$ Update: Endre Csóka recently claimed an unpublished upper bound $2 \sqrt{2}$ using topology.
} 


\section{Proof of Theorem 1.2}

The idea is to find an $x \in \mathbb{R}$ such that the transversal we look for satisfies the condition

$$
a_{i} \in a_{i-1}+[x, x+1] .
$$

With this strategy the condition $\left|z_{i}-z_{j}\right| \leq 1$ is automatically guaranteed. The question is whether there is an $x \in \mathbb{R}$, which admits a transversal $a_{0}, \ldots, a_{n}$ satisfying condition (3) for every $i \in[n]$.

We analyze what happens when this strategy is followed. A partial transversal is just a selection of $a_{i} \in A_{i}$ for $i=0, \ldots, h, h \in[n]$. We call it $x$-good if it satisfies condition (3) for every $i \in[h]$. As a first step, $a_{1}$ is to be chosen from the interval $J_{1}=a_{0}+[x, x+1]=[x, x+1]$, and $J_{1} \cap A_{1}$ is nonempty because of condition (ii). Thus, $a_{0}, a_{1}$ is an $x$-good partial transversal for any $a_{1} \in J_{1} \cap A_{1}$.

It is easy to see that $x$-good transversals exist for every $x \in \mathbb{R}$ and $h \in[n-1]$. To construct such partial transversals we define $J_{i}$ recursively as follows. Given $J_{i}$ for some $i \in[n-1]$ and a fixed $x \in \mathbb{R}$, we let

$$
J_{i+1}=\left(J_{i} \cap A_{i}\right)+[x, x+1]=\bigcup\left\{[a+x, a+x+1]: a \in J_{i} \cap A_{i}\right\} .
$$

A routine induction, based on condition (ii), shows that $J_{i}$ is a closed interval of length at least one for every $i \in[n]$, and so $J_{i}$ intersects $A_{i}$ if $i \in[n-1]$. The intervals $J_{i}$ of course depend on $x$, and we write $J_{i}(x)=\left[L_{i}(x), R_{i}(x)\right]$ to express this dependence. The definition of $J_{i}(x)$ implies that

$$
\begin{aligned}
& L_{i+1}(x)=x+\min \left\{a \in A_{i}: a \geq L_{i}(x)\right\} \\
& R_{i+1}(x)=x+1+\max \left\{a \in A_{i}: a \leq R_{i}(x)\right\} .
\end{aligned}
$$

Note that both $L_{i}(x)$ and $R_{i}(x)$ are increasing functions of $x$ satisfying $R_{i}(x)-L_{i}(x) \geq 1$. Also, $L_{i+1}(x) \geq x+L_{i}(x)$ implying via an easy induction that $L_{i}(x)$ and then $R_{i}(x)$ tends to infinity as $x \rightarrow \infty$. A similar argument shows that $\lim R_{i}(x)=\lim L_{i}(x)=-\infty$ as $x \rightarrow-\infty$. It is also clear that if $a_{h} \in J_{h}(x) \cap A_{h}$ for some $x \in \mathbb{R}$, then there exists an $x$-good partial transversal $a_{0}, a_{1}, \ldots, a_{h}$.

To complete the proof of Theorem 1.2 we only have to show that there is an $x \in \mathbb{R}$ such that $J_{n}(x) \cap A_{n}$ is non-empty, that is, $s \in J_{n}(x)$. Actually we prove more:

Lemma 2.1. For every $h=1, \ldots, n$ the intervals $J_{h}(x)(x \in \mathbb{R})$ cover every point of $\mathbb{R}: \bigcup_{x \in \mathbb{R}} J_{h}(x)=$ $\mathbb{R}$.

Proof. First we claim that $L_{i}$ is a left continuous function for every $i \in[n]$. This is evident for $i=1$, so we assume that $L_{i}$ is left continuous and proceed to prove that $L_{i+1}$ is also left continuous. In view of (4) it amounts to checking that

$$
f(x)=\min \left\{a \in A_{i}: a \geq L_{i}(x)\right\}
$$

is a left continuous function of $x$. Let $x \in \mathbb{R}$ be arbitrary; we have to show that $x_{j}<x, x_{j} \rightarrow x$ implies $f\left(x_{j}\right) \rightarrow f(x)$. It is clear that if $\left[L_{i}(u), L_{i}(x)\right) \cap A_{i}=\emptyset$ for some $u<x$, then $f$ is constant on the interval $[u, x]$ and $f\left(x_{j}\right)=f(x)$ for $x_{j} \geq u$.

Otherwise there is a sequence $a_{j} \in A_{i}$ such that

$$
L_{i}\left(x_{j}\right) \leq f\left(x_{j}\right)=a_{j}<L_{i}(x) .
$$

Here $L_{i}\left(x_{j}\right) \rightarrow L_{i}(x)$ by the left continuity of $L_{i}$. Accordingly,

$$
L_{i}(x)=\lim _{j \rightarrow \infty} a_{j} \in A_{i}
$$

because $A_{i}$ is closed. It follows that $f\left(x_{j}\right) \rightarrow L_{i}(x)=f(x)$. The left continuity of $f$ at $x$ is thus established. 
Similarly, $R_{i}$ is a right continuous function for every $i \in[n]$. To complete the proof of the lemma, consider any point $p \in \mathbb{R}$ and define

$$
\begin{aligned}
L^{p}=L_{h}^{p} & =\left\{x \in \mathbb{R}: L_{h}(x) \leq p\right\}, \\
R_{p}=R_{h}^{p} & =\left\{x \in \mathbb{R}: R_{h}(x) \geq p\right\} .
\end{aligned}
$$

Here neither $L^{p}$ nor $R^{p}$ is empty since $\lim _{x \rightarrow-\infty} L_{h}(x)=-\infty$ and $\lim _{x \rightarrow \infty} R_{h}(x)=\infty$. The continuity and monotonicity properties of the functions $L_{h}$ and $R_{h}$ imply that both $L^{p}$ and $R^{p}$ are closed sets. Further, $L^{p} \cup R^{p}=\mathbb{R}$, as $x \notin L^{p}$ implies $L_{h}(x)>p$ and $x \notin R^{p}$ implies $R_{h}(x)<p$, and then $R_{h}(x)<p<L_{h}(x)$, which is impossible.

Now if two closed sets cover the connected space $\mathbb{R}$, then they have a point in common. So there is an $x_{0} \in L^{p} \cap R^{p}$, and then $L_{h}\left(x_{0}\right) \leq p \leq R_{h}\left(x_{0}\right)$, so $p \in J_{h}\left(x_{0}\right)$. Thus every $p \in \mathbb{R}$ is contained in some $J_{h}(x)$.

Remark. The proof of Theorem 1.1, which is an algorithm of complexity $O\left(\mathrm{~nm}^{3}\right)$ can be modified to give another (algorithmic) proof of Theorem 1.2. But the above proof can be turned into an approximation algorithm the following way. By the remark at the end of the introduction we may assume that $s=0$. Note that for $x=-1$ no interval $J_{h}(x)$ contains a positive number, and similarly, for $x=0$ no interval $J_{h}(x)$ contains a negative one. Using binary search, after $k$ iteration, one finds an $x \in[-1,0]$ that is within distance $2^{-k}$ of the solution.

\section{Proof of Theorem 1.5}

We begin with an informal description of the construction. We fix a large enough integer $m$. The gridlike sequence consists of $2 m+2$ sets $A_{0}, \ldots, A_{2 m+1}$, where $A_{0}=A_{2 m+1}=\{0\}$. Recall that in this case $0 \in Z$. Each other $A_{i}$ is the union of sets $N_{i}, S_{i}, E_{i}, W_{i}$ (corresponding to North, South, East, and West) plus four corners $Q_{i}^{N E}, Q_{i}^{S E}, Q_{i}^{N W}, Q_{i}^{S W}$, see the figures below. The sets $A_{i}$ are symmetric about the $x$ and $y$ axes. The sets $A_{1}, \ldots, A_{m}$ make up the first part of the construction. For $i \in\{m+1, \ldots, 2 m\}$, $A_{i}$ is the refection of $A_{2 m+1-i}$ about the line $x=y$. In particular, $A_{m}=A_{m+1}$.

The main characters in our construction are a square $X$ and segments $G_{i}$ of changing length that are either horizontal or vertical. The Minkowski sum $X+G_{i}$ is a hexagon shown on Figures 1 and 2. Its vertical and horizontal sides (or vertices) are drawn with heavy lines, its oblique sides with thin segments. The horizontal and vertical sides (or vertices) are extended to the sets $N_{i}, S_{i}, E_{i}, W_{i}$.

The main step of the proof is to show that an optimal tranversal $a_{0}, \ldots, a_{2 m+1}$ has no point in the corners and that it does not visit the same region ( $N$ or $S$ or $E$ or $W$ ) twice. More precisely, if $a_{i} \in N_{i}$ and $a_{j} \in N_{i}$ for some $i<j$, then $a_{h} \in N_{h}$ for all $h \in\{i, \ldots, j\}$, and the same for the components of type $S, E, W$.

Formally we define the various components of $A_{i}$ for $1 \leq i \leq m$ as

$$
\begin{aligned}
N(a, b) & =\{(x, y):-a \leq x \leq a, y \geq b\}, \\
S(a, b) & =\{(x, y):-a \leq x \leq a, y \leq b\}, \\
E(c, d) & =\{(x, y): x \geq c,-d \leq y \leq d\}, \\
W(c, d) & =\{(x, y): x \leq c,-d \leq y \leq d\},
\end{aligned}
$$

and

$$
\begin{aligned}
Q^{N W}(e, f) & =\{(x, y): e \geq x, f \leq y\} \\
Q^{N E}(e, f) & =\{(x, y): e \leq x, f \leq y\} \\
Q^{S W}(e, f) & =\{(x, y): e \geq x, f \geq y\} \\
Q^{S E}(e, f) & =\{(x, y): e \leq x, f \geq y\}
\end{aligned}
$$




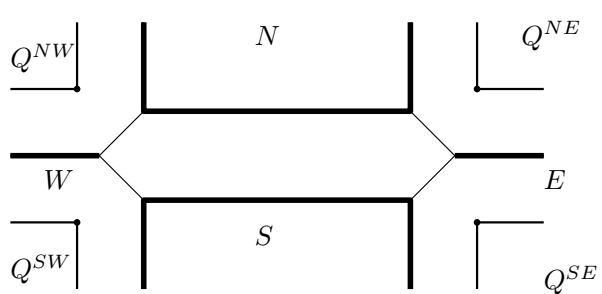

$A_{1}$

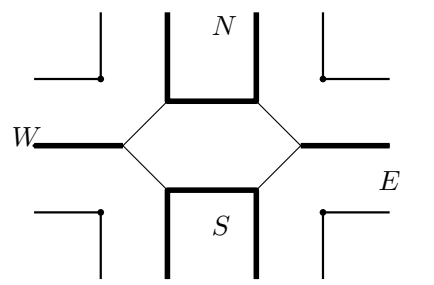

$A_{2 m / 3}$

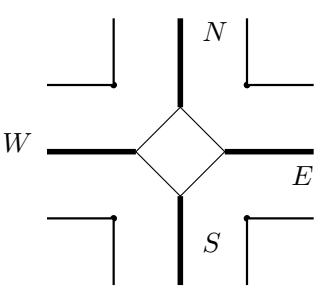

$A_{m}$

Figure 1. Some $A_{i}$ from phase 1

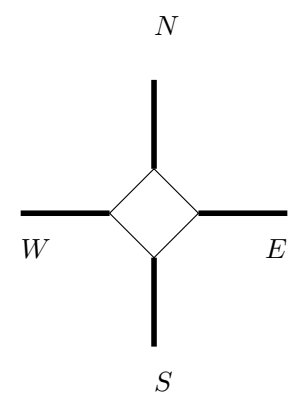

$A_{m+1}$

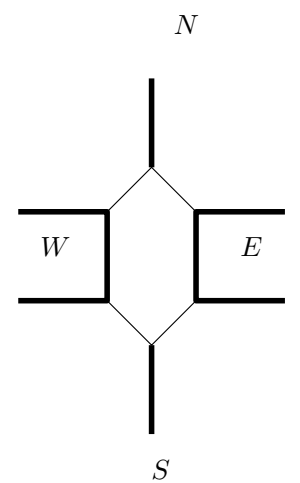

$A_{4 m / 3}$

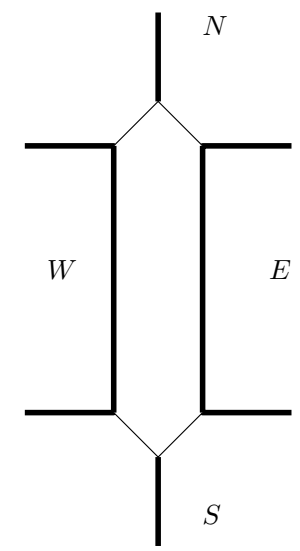

$A_{2 m+1}$

Figure 2. Some $A_{i}$ from phase 2, corners suppressed

with appropriately chosen parameters $a, b, c, d, e, f$ depending on $i$. We call $V(a, b)=N(a, b) \cup S(a,-b)$ and $H(c, d)=E(c, d) \cup W(-c, d)$ the vertical and horizontal parts, and the set

$$
Q(e, f)=Q^{N W}(-e, f) \cup Q^{N E}(e, f) \cup Q^{S W}(-e,-f) \cup Q^{S E}(e,-f)
$$

the union of the corners. Set first $A_{1}=V_{1} \cup H_{1} \cup Q_{1}$, where

$$
V_{1}=V(3,1), \quad H_{1}=H(4,0), \quad Q_{1}=Q(4.5,1.5) .
$$

Here the values 4.5 and 1.5 are chosen so that $A_{1}$, and later all other $A_{i}$ satisfy condition (ii). Next, for $i=2, \ldots, m$ we define $A_{i}=V_{i} \cup H_{i} \cup Q_{i}$, where

$$
V_{i}=V(3-(i-1) \delta, 1), H_{i}=H(4-(i-1) \delta, 0), Q_{i}=Q(4.5-(i-1) \delta, 1.5)
$$

with $\delta=3 /(m-1)$. Note that $A_{m}$ is made up of four halflines and the four corners. For $i=m+1, \ldots, 2 m$ we let $A_{i}$ be the reflected copy of $A_{2 m+1-i}$ about the line $x=y$. It is easy to check that $A_{0}, A_{1}, \ldots, A_{2 m+1}$ is a grid-like sequence for $n=2 m+1$.

Claim 3.1. There is a transversal $T$ with $D(T) \leq 4 \sqrt{2-\sqrt{3}}$. 


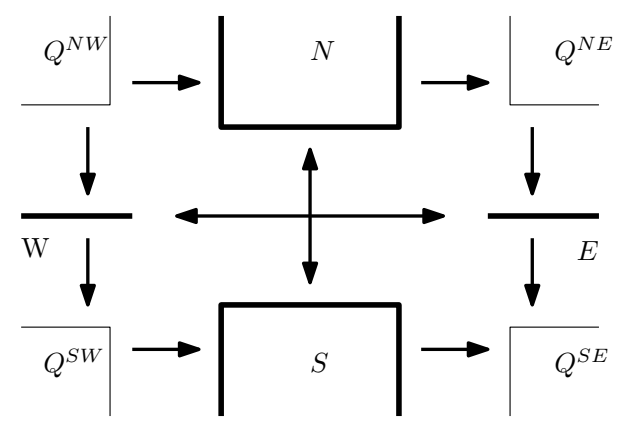

FiguRE 3. Forbidden jumps

Proof. Consider the transversal $T=\left\{a_{0}, \ldots, a_{2 m+1}\right\}$ where

$$
a_{i}=\left(\frac{m-i}{m-1}(2 \sqrt{3}-3), 1\right), \quad a_{m+i}=\left(1, \frac{i-1}{m-1}(2 \sqrt{3}-3)\right)
$$

for $i=1, \ldots, m$. Then $Z$ is an equilateral triangle whose vertices are

$$
z_{1}=(2 \sqrt{3}-3,1), \quad z_{m+1}=(1,-1), \quad z_{2 m+1}=(-1,3-2 \sqrt{3}),
$$

and $\operatorname{diam} Z=4 \sqrt{2-\sqrt{3}}$.

Fix an optimal transversal $T=\left\{a_{0}, \ldots, a_{2 m+1}\right\}$, such a transversal exists by compactness. Write $z_{i}=\left(x_{i}, y_{i}\right)$. As $0 \in Z$, the above claim implies $\left\|z_{i}\right\| \leq 4 \sqrt{2-\sqrt{3}}$.

We say that $a_{j}$ jumps if $a_{j-1}$ is in one type of component in $A_{j-1}$ but $a_{j}$ is in another type in $A_{j}$. Then $z_{j}$ is called the corresponting jump. For instance $a_{j}$ jumps if $a_{j-1} \in Q_{j-1}^{N W}$ but $a_{j}$ is in $N_{j}$ or in $W_{j}$. The important property is that $\left\|z_{j}\right\|$ is large when $z_{j}$ is a jump. Therefore the structure of the sequence of jumps is rather restricted.

Assume by symmetry that $a_{1} \in N_{1}$. Then $z_{1}=a_{1}$ and $y_{1} \geq 1$. Similarly, we may assume that $a_{2 m} \in E_{2 m}$. Then $z_{n}=-a_{n-1}$ and $x_{n} \leq-1$.

Fact 1. For every $i=1, \ldots, 2 m+1, y_{i} \geq-1.1$ and $x_{i} \leq 1.1$. Proof: Otherwise the $y$ component of $z_{1}-z_{i}$ or the $x$-component of $z_{i}-z_{n}$ is larger than 2.1 and then diam $Z>2.1$, which contradicts Claim 3.1 and the optimality of $T$.

This implies that the jumps $Q^{N W} \rightarrow N, N \rightarrow Q^{N E}, Q^{S W} \rightarrow S, S \rightarrow Q^{S E}$ and $W \rightarrow E$ are forbidden, and so are the jumps $Q^{N W} \rightarrow W, W \rightarrow Q^{S W}, Q^{N E} \rightarrow E, E \rightarrow Q^{S E}$ and $N \rightarrow S$, see Figure 3. In particular, $Q^{S E}$ and $Q^{N W}$ is never visited by $T$, because all other components $T$ could jump from $Q^{N W}$ into or from which $T$ could jump into $Q^{S E}$ are too far away. Moreover, as indicated on Figure 3, there cannot be $E \rightarrow W$ or $S \rightarrow N$ jumps either. Indeed, suppose that there is a jump from some $E_{i}$ to $W_{i+1}$. To get back to the point $a_{2 m} \in E_{2 m}$ there must be another jump to $E_{j}$ for some $j>i+1$. But then $x_{i+1} \leq-2$ and $x_{j} \geq 1-\delta$, yielding $\left\|z_{j}-z_{i+1}\right\| \geq 3-\delta$, a contradiction. A similar argument applies to an $S \rightarrow W$ jump.

We say that a pair of jumps is opposite if either (a) one is $N \rightarrow E$ or $W \rightarrow S$ and the other is $E \rightarrow N$ or $S \rightarrow W$, or (b) one is $N \rightarrow W$ or $E \rightarrow S$ and the other is $W \rightarrow N$ or $S \rightarrow E$.

Fact 2. There cannot be an opposite pair of jumps if $m$ is large enough. Proof: If $z_{i}$ and $z_{j}$ are opposite jumps, then $\left|x_{j}-x_{i}\right| \geq 2-2 \delta$ and $\left|y_{j}-y_{i}\right| \geq 2-2 \delta$. Thus ||$z_{j}-z_{i}||>2.8$ if $\delta$ is small enough, a contradiction.

Lemma 3.2. There is a single jump and it goes from $N_{j-1}$ to $E_{j}$ for some $j=2, \ldots, 2 m$. 

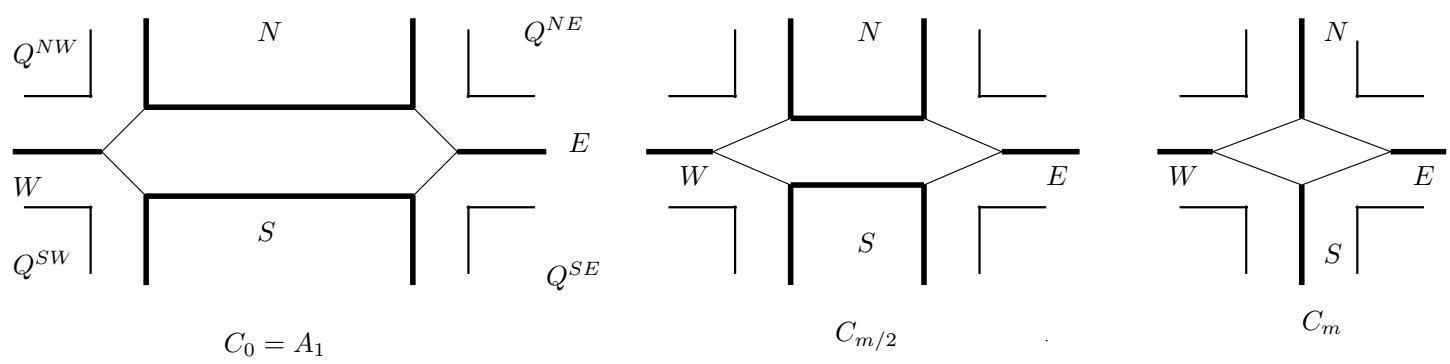

Figure 4. $C_{0}, C_{m / 2}, C_{m}$, the rhombus $R$ is drawn with thin segments

Proof. There must be a jump since $a_{1} \in N_{1}$ and $a_{2 m} \in E_{2 m}$. Assume that the first jump is $z_{j}$, that is $a_{i} \in N_{i}$ for $i=1, \ldots, j-1$ but $a_{j} \notin N_{j}$. As we have seen, $a_{j}$ is either in $E_{j}$ or in $W_{j}, Q_{j}^{S W}$ and $Q_{j}^{S E}$ too far away.

Suppose first that $a_{j} \in W_{j}$, we will see that it leads to a contradiction. Note that $z_{j}$ is fairly large: $x_{j} \leq-1+\delta$ and $y_{j} \leq-1+\delta$. Also, there must be a further jump, say $z_{k}$ meaning that $a_{i} \in W_{i}$ for $i=j, \ldots, k-1$ but $a_{k} \notin W_{k}$. So $a_{k}$ is in $S_{k}$ or in $N_{k}$ since $Q_{k}^{N E}$ is too far away if $m$ is large enough. Now $a_{2 m} \in E_{2 m}$ which cannot be reached from $a_{k}$ without creating an opposite pair: along the way there is an $S \rightarrow E$ jump or a $N \rightarrow E$ one. A contradiction.

We can conclude that the first jump goes from $N_{j-1}$ to $E_{j}$.

If there is a further jump, then the next jump, $z_{k}$ say, must go from $E_{k-1}$ to $Q_{k}^{N E}$ or $S_{k}$ or $N_{k}$, as $Q_{k}^{S W}$ is too far away. Here $N_{k}$ is excluded as then $z_{j}, z_{k}$ are opposite. If $a_{k} \in Q_{k}^{N E}$, then $\left\|z_{k}-z_{j}\right\|>$ $\left|y_{k}-y_{j}\right| \geq 2.5-\delta$. Assume finally that $a_{k} \in S_{k}$. Then, again, $a_{2 m} \in E_{2 m}$ cannot be reached from $a_{k} \in S_{k}$ without using an opposite pair: along the way there is an $S \rightarrow E$ jump or an $W \rightarrow N$ one.

Let $z_{j}$ be the single jump along the way. Note that $x_{j} \geq 1-\delta$ and $y_{j} \leq-1+\delta$. This leads to a simple minimisation problem. Given points $z_{1}, z_{j}, z_{n} \in \mathbb{R}^{2}$, with $y_{1} \geq 1, x_{j} \geq 1-\delta, y_{j} \leq-1+\delta$ and $x_{n} \leq-1$, find the minimum diameter of the triangle formed by these points. As $\delta$ tends to 0 , the unique solution to this problem converges to the equilateral triangle specified in the proof of Claim 3.1. Thus, $D(T)>4 \sqrt{2-\sqrt{3}}-\varepsilon$ for $m \geq m_{0}(\varepsilon)$. This completes the proof of Theorem 1.5 for odd values of $n$. For even values of $n$ some straightforward modifications are needed, which are left to the reader.

\section{Sketch of the Proof of Theorem 1.6}

In this construction $m$ is a large integer again, and the grid-like sequence of sets is $C_{-1}, C_{0}, \ldots$, $C_{6 m+1}, C_{6 m+2}$, where $C_{-1}=C_{6 m+2}=\{(0,0)\}$. Further $C_{0}=A_{1}, C_{6 m+1}=A_{2 m}$, where $A_{1}, A_{2 m}$ come from the previous construction to guarantee that $\left|y_{0}\right| \geq 1$ and $\left|x_{6 m+1}\right| \geq 1$ with the previous notation $z_{i}=a_{i}-a_{i-1}=\left(x_{i}, y_{i}\right)$.

The main characters are a rhombus $R$ (instead of the square $X$ ) and the same segments $G_{i}$. The length of the shorter diagonal of the rhombus is $\sqrt{2}$, its sides are of length $\sqrt{2+\sqrt{2}}$, and its centre is the origin. But this time rotated copies of $R$ are needed, so let $R(\alpha)$ stand for the rotated copy of $R$; rotated by angle $\alpha$ in clockwise direction around its centre.

The first $m$ sets $C_{1}, \ldots, C_{m}$ come from the Minkowski sum of $R$ and the previous horizontal $G_{i}$ : This sum is a hexagon again and the components $N_{i}, S_{i}, E_{i}, W_{i}$ are just extended from the horizontal and 

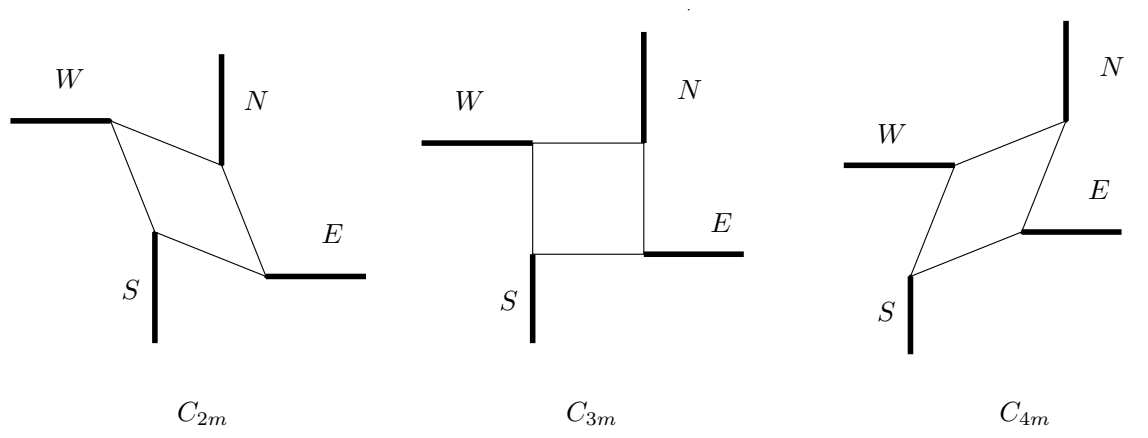

FiguRE 5. Deformation of the rhombus from $C_{2 m}$ to $C_{4 m}$, corners suppressed

vertical sides of this hexagon the same way as in Theorem 1.5. The corners are at the same distance from the horizontal and vertical components as before. See Figure 4 . The last $C_{5 m+1} \ldots, C_{6 m}$ sets come from the Minkowski sum of $R(\pi / 2)$ and the corresponding vertical segments $G_{i}$ analogously.

Note that $N_{m}, S_{m}, E_{m}, W_{m}$ are halflines, and they remain halflines in all $C_{i}$ with $i \in\{m, \ldots, 5 m\}$. The sets $C_{m+1}, \ldots, C_{2 m}$ come from gradually rotated copies of $R$. Then the rhombus $R(\pi / 4)$ that defines $C_{2 m}$ is gradually deformed to a square (of side legth $\sqrt{2}$ ) in $C_{3 m}$, which is further deformed to the rhombus $R(-\pi / 4)$ in $C_{4 m}$, see Figure 5. Then $R(-\pi / 4)$ rotates back to $R(-\pi / 2)=R(\pi / 2)$ in $C_{5 m}$.

The proof that this construction gives $D(T)>1+\sqrt{2}-\varepsilon$ is based on ideas similar to those used in Theorem 1.5: First one shows that an optimal transversal $T$ has no point in the corners, and second, that $T$ does not visit the same type component $N, S, E, W$ twice. We omit the details.

Acknowledgements. IB was supported by ERC Advanced Research Grant no 267165 (DISCONV) and by National Research, Development and Innovation Office NKFIH Grants K 111827 and K 116769. ECs was supported by ERC grants 306493 and 648017 and by Marie Curie Fellowship, grant No. 750857. GyK was partially supported by bilateral research grant TÉT 12 MX-1-2013-0006. GT was supported by the National Research, Develpoment and Innovation Office NKFIH Grant K-111827.

\section{REFERENCES}

[1] I. Bárány, V. S. Grinberg. Block partitions of sequences, Israel J Math., 206 (2015), 155-164.

[2] M. Lucertini, Y. Perl, B. Simeone. Most uniform path partitiong and its use in image processing, Discrete Applied Mathematics, 42 (1993), 227-256.

Imre Bárány

Alfréd Rényi Institute of Mathematics,

Hungarian Academy of Sciences

13 Reáltanoda Street Budapest 1053 Hungary

and

Department of Mathematics

University College London

Gower Street, London, WC1E 6BT, UK

barany.imre@renyi.mta.hu

Endre Csóka

Alfréd Rényi Institute of Mathematics, Hungarian Academy of Sciences 
13 Reáltanoda Street Budapest 1053 Hungary

csokaendre@gmail.com

Gyula Károlyi

Alfréd Rényi Institute of Mathematics,

Hungarian Academy of Sciences

13 Reáltanoda Street Budapest 1053 Hungary

and

Institute of Mathematics

Eötvös University

1/C Pázmány P. sétány Budapest 1117 Hungary

karolyi.gyula@renyi.mta.hu

Géza Tóth

Alfréd Rényi Institute of Mathematics,

Hungarian Academy of Sciences

13 Reáltanoda Street Budapest 1053 Hungary

toth.geza@renyi.mta.hu 